Japanese Journal of Applied Physics, 48 (2) (2009) 020223

\title{
Electron Spin Resonance of X-ray-Irradiated CdS-Doped Glass before Heat Treatment
}

Tadaki Miyoshi ${ }^{*}$, Kouto Migita ${ }^{1}$, Masakatsu Tamechika ${ }^{2}$, Nobuko Tokuda ${ }^{2}$, and Teruhisa Kaneda $^{3}$

Division of M aterials Engineering, Graduate School of Science and Engineering, Yamaguchi U niversity, Tokiwadai, U be, Yamaguchi 755-8611, Japan

${ }^{1}$ Division of Chemistry, Graduate School of Science and Engineering, Yamaguchi University, Yoshida, Yamaguchi 753-8512, J apan

${ }^{2}$ Department of Organ Anatomy, Yamaguchi U niversity Graduate School of M edicine, M inami-K ogushi, U be, Yamaguchi 755-8505, J apan

${ }^{3}$ U be National College of Technology, Tokiwadai, U be, Yamaguchi 755-8555, J apan

(Received October 28, 2008; accepted December 22, 2008)

The electron spin resonance (ESR) spectrum has been measured in X-ray-irradiated CdS-doped glass before heat treatment to examine the possibility of using such glass as a dosimetric material. No ESR signal is observed before X-ray irradiation, but it was observed after X-ray irradiation. The intensity of the ESR signal of the glass before heat treatment is higher than that after heat treatment. The thermal stability of the ESR signal of the former glass is slightly better than that of the latter. Therefore, the glass before heat treatment has better properties than that after heat treatment.

*E-mail address: tmiyoshi@yamaguchi-u.ac.jp 
Alanine is used for radiation dosimetry using electron spin resonance (ESR). Recently, Hassan et al. ${ }^{1)}$ reported that bioglass $\left(45 \% \mathrm{SiO}_{2}, 24.5 \% \mathrm{CaO}, 24.5 \% \mathrm{Na}_{2} \mathrm{O}\right.$, and $\left.6 \% \mathrm{P}_{2} \mathrm{O}_{5}\right)$ is tenfold more sensitive than commercial alanine dosimeters. In a previous paper, ${ }^{2)}$ we reported that an ESR signal was observed in X-ray-irradiated CdS-doped glass, Y44, which contains CdS nanocrystals in silicate glass, and that Y-44 is more sensitive than bioglass and the alanine dosimeter. The intensity of the ESR signal increases with decreasing size of CdS nanocrystals in laser-irradiated CdS-doped glass. ${ }^{3)}$ Therefore, Xray-irradiated CdS-doped glass containing smaller nanocrystals is anticipated to be more sensitive than Y-44.

Y-44 is a filter glass obtained after heat treatment. Cd and S are dissolved in silicate glass at $1300-1400^{\circ} \mathrm{C}$. After the glass is cooled, CdS nanocrystals are formed by heat treatment at $575-750^{\circ} \mathrm{C}{ }^{4)}$ The size of CdS nanocrystals in the filter glass increases with increasing temperature and time of heat treatment. Although $\mathrm{Cd}$ and $\mathrm{S}$ mainly exist as dissolved ions, a small number of smaller nanocrystals of CdS may also be formed in CdS-doped glass before heat treatment. In fact, Yanagawa et al.5) observed 1-nm-order nanocrystals of CdSSe in CdSSe-doped glass before heat treatment, using a transmission electron microscope. Smaller nanocrystals of CdS are considered to exist in CdS-doped glass before heat treatment. Here, we report the ESR spectrum of CdS-doped glass before heat treatment, which may contain nanocrystals smaller than those in Y-44. The intensity and the thermal stability of the ESR signal of the CdS-doped glass before heat treatment were measured and compared with those of Y-44.

The sample investigated was mainly CdS-doped filter glass, Asahi Y-02, which is a glass for filters before heat treatment. Commercial filter glass, Y-44, is the glass form of Y-02 after heat treatment. The size of the samples was $2 \mathrm{~mm}$ in width, $2.5 \mathrm{~mm}$ in thickness and $10 \mathrm{~mm}$ in length. The concentration of CdS was approximately $0.4 \mathrm{wt} \%{ }^{6}$ ) The size of CdS nanocrystals in Y-44 was approximately $3 \mathrm{~nm} .{ }^{3)}$ The glass composition was $70 \%$ $\mathrm{SiO}_{2}, 10 \% \mathrm{Na}_{2} \mathrm{O}, 10 \% \mathrm{ZnO}, 6 \% \mathrm{~K}_{2} \mathrm{O}$, and $3 \% \mathrm{~B}_{2} \mathrm{O}_{3} .{ }^{6)}$ We also measured the ESR spectra of Y-44 and undoped glass, Y-0, which does not contain CdS, for comparison.

The glass samples were exposed to X-rays from an X-ray source (Hitachi Medico MBR1520R, W target, $150 \mathrm{kV}, 20 \mathrm{~mA}$ ) at $300 \mathrm{~K}$. Low-energy X-rays were eliminated using a 
filter, which was composed of an $\mathrm{Al}$ plate and a $\mathrm{Cu}$ plate. The filter mainly used was composed of an $\mathrm{Al}$ plate with a thickness of $0.5 \mathrm{~mm}$ and a $\mathrm{Cu}$ plate with a thickness of $0.1 \mathrm{~mm}$, and consequently, the effective X-ray energy was $48 \mathrm{keV}$.

ESR spectra were measured using an X-band ESR spectrometer (Bruker ELEXSYS E$500)$ at $300 \mathrm{~K}$ with a cylindrical cavity operated at $9.76 \mathrm{GHz}$ with a $100 \mathrm{kHz}$ modulation frequency. The microwave power was $10 \mathrm{~mW}$, since the intensity of ESR signals tended to saturate at $20 \mathrm{~mW}$. The modulation amplitude was $0.1 \mathrm{mT}$ for Y-02 and Y-44, and $0.5 \mathrm{mT}$ for Y-0. The response time constant was $0.16 \mathrm{~s}$ with a field-sweeping rate of $20 \mathrm{mT} / 84 \mathrm{~s}$. Transmission spectra were measured using a spectrophotometer (JASCO V-550) at $300 \mathrm{~K}$.

Isochronal annealing was performed by heating irradiated samples at temperatures varying from $100^{\circ} \mathrm{C}$ to $250^{\circ} \mathrm{C}$. The annealing time was $15 \mathrm{~min}$.

Figure 1 shows the transmission spectra of unirradiated CdS-doped glasses, Y-02 and Y-44, and undoped glass, Y-0, at $300 \mathrm{~K}$. Y-44 contains nanocrystals of CdS and absorbs light of wavelength shorter than about $430 \mathrm{~nm}$. In contrast, Y-02 is considered to contain a small number of smaller nanocrystals of CdS and slightly absorbs visible light.

Figure 2 shows ESR spectra of X-ray irradiated CdS-doped glasses, Y-02 and Y-44, at $300 \mathrm{~K}$. The ESR spectrum for the CdS-doped glass is characterized by two main signals: $g=2.01$ (around $347 \mathrm{mT}$ ) and $g=1.99$ (around $351 \mathrm{mT}$ ). The signal at $g=1.99$ may be ascribed to a trapped electron on a $\mathrm{Cd}^{2+}$ ion in the glass matrix. ${ }^{2)}$ The intensity of the signal at $g=1.99$ for $\mathrm{Y}-02$ is larger than that for Y-44. Since Y-02 is not heat treated, $\mathrm{Cd}$ mainly exists as dissolved ions. Consequently, the concentration of $\mathrm{Cd}^{2+}$ ions in $\mathrm{Y}-02$ glass is higher than that in Y-44 glass. This explains why the signal at $g=1.99$ for Y-02 is higher than that for $\mathrm{Y}-44$; that is, $\mathrm{Y}-02$ is more sensitive than Y-44. The signal at $g=2.01$ is similar to that in bioglass. ${ }^{1)}$ Therefore, this signal may be ascribed to the oxygen hole center. The hole centers are considered to be located in the interface between CdS nanocrystals and glass. ${ }^{3)}$ Since the number of CdS nanocrystals in Y-02 is probably less than that in Y-44, the number of hole centers is also probably less than that in Y-44. This explains why the signal at $g=2.01$ for $\mathrm{Y}-02$ is lower than that for Y-44. The ESR signal was not observed in unirradiated glasses.

Figure 3 shows the dose response of ESR intensities for Y-02, Y-44, and the alanine 
dosimeter. The peak-to-peak intensities of the ESR signals at $g=1.99$ and $g=2.01$ are plotted as a function of X-ray dose. The dose response shows slight sublinearity for doses higher than about $40 \mathrm{~Gy}$. The intensity of the ESR signal at $g=1.99$ for Y-02, Y-02(e), is higher than that for Y-44, Y-44(e), and that for the alanine dosimeter. The intensity of the ESR signal at $g=2.01$ for $\mathrm{Y}-44, \mathrm{Y}-44(\mathrm{~h})$, is almost the same as that at $g=1.99$ for Y-02, Y-02(e). However, the ESR signal at $g=1.99$ is suited to dosimetry, since distortion of the signal at $g=1.99$ is less than that at $g=2.01$ when the modulation amplitude is increased to enable the measurement of weak signals. While the distortion of the signal at $g=2.01$ is observed for the modulation amplitude of $0.5 \mathrm{mT}$, that at $g=1.99$ is not observed for $0.5 \mathrm{mT}$ and $1 \mathrm{mT}$. The intensity of the ESR signal at $g=1.99$ is proportional to the modulation amplitude up to $1 \mathrm{mT}$.

Figure 4 shows the ESR spectrum of X-ray irradiated undoped glass, Y-0, at $300 \mathrm{~K}$. The signal at $g=1.99$ is not observed. Electrons may be trapped by other defects. Since the $g$ value of the trapped electron may be near 2.00, the ESR signal may be overlapped with the signal at $g=2.01$. Since the intensity of the ESR signal of Y-0 is lower than those of Y-02 and Y-44, a higher modulation amplitude is used to observe the ESR signal of $\mathrm{Y}-0$, and the exposure time of X-rays is also increased in order to increase the signal intensity: $0.5 \mathrm{mT}$ and $40 \mathrm{~Gy}$ for Y-0, and $0.1 \mathrm{mT}$ and $10 \mathrm{~Gy}$ for Y-02 and Y-44. The real intensity of the signal of Y-0 is estimated to be about 1/15 that of Y-44, if these samples are measured under the same experimental conditions.

The isochronal annealing results for Y-02 and Y-44 at temperatures ranging from $100^{\circ} \mathrm{C}$ to $250^{\circ} \mathrm{C}$ are shown in Fig. 5 . The normalized intensities of the ESR signals at $g=1.99$ are plotted as a function of annealing temperature. The ESR signals vanish at $200^{\circ} \mathrm{C}$. This indicates that trapped electrons are detrapped at $200^{\circ} \mathrm{C}$; this is consistent with the result that the thermoluminescence glow peak is observed near $200^{\circ} \mathrm{C} .^{7,8)}$ The data in Fig. 5 do not follow Arrhenius' law. This is attributable to the fact that the traps have distributed energy. The distribution of traps was confirmed by analyzing the glow curve of thermoluminescence. ${ }^{8)}$

Figure 6 shows the fading characteristics of Y-02, Y-44, and Y-0 at $300 \mathrm{~K}$. The intensities of the ESR signals at $g=1.99$ are plotted for Y-02 and Y-44. On the other hand, 
the intensity of the ESR signal at $g=2.01$ is plotted for $\mathrm{Y}-0$, since the ESR signal at $g=1.99$ is not observed for Y-0. The intensities of the ESR signals at $g=2.01$ for Y-02 and Y-44 decrease similarly to those at $g=1.99$. The ESR intensities are normalized. The intensity ratios are as follows: 2 (Y-02), 1 (Y-44), and 0.07 (Y-0). Since the ESR intensity of Y-0 is lower than those of Y-02 and Y-44, it is difficult to determine the precise intensity of Y-0 after it has been left for long periods. The signal intensities of Y-02 and Y-44 decrease rapidly at an earlier stage in the time dependence of signal intensities, and then decrease gradually. This is attributable to the fact that the traps have distributed energy. Electrons in shallower traps are detrapped within one or two months after X-ray irradiation. On the other hand, electrons in deeper traps are negligibly detrapped. The ESR signal of Y-0 decreases rapidly. This indicates that traps in Y-0 are shallower than those in Y-02 and Y-44, and the defects associated with CdS may trap electrons for longer periods. The thermal stability of the ESR signal of Y-02 is slightly better than that of Y-44.

The minimum detectable dose is approximately $300 \mathrm{mGy}$ for Y-02 and $500 \mathrm{mGy}$ for Y-44, when the modulation amplitude is $0.5 \mathrm{mT}$. The minimum detectable dose of Y02 decreases with increasing modulation amplitude, since the distortion of the signal at $g=1.99$ for Y-02 is less than that at $g=2.01$ for Y-44.

In summary, ESR signals have been observed in X-ray-irradiated CdS-doped glasses before and after heat treatment, Y-02 and Y-44, respectively. The ESR signal associated with trapped electrons in $\mathrm{Y}-02$ is higher than that in $\mathrm{Y}-44$, that is, $\mathrm{Y}-02$ is more sensitive than Y-44. The thermal stability of the ESR signal of Y-02 is slightly better than that of Y-44. These results indicate that Y-02 may be used as a more sensitive dosimeter than Y-44 or other dosimeters such as bioglass and alanine.

Transmission spectra were measured using the spectrophotometer in the laboratory of Professor K. Kasatani. The authors are grateful to Professor K. Kasatani. 


\section{References}

1) G. M. Hassan, M. A. Sharaf, and O. S. Desouky: Radiat. Meas. 38 (2004) 311.

2) T. Miyoshi, K. Migita, M. Tamechika, N. Tokuda, T. Fukumoto, and T. Kaneda: Jpn. J. Appl. Phys. 45 (2006) 7105.

3) T. Miyoshi, H. Matsuki, and N. Matsuo: Jpn. J. Appl. Phys. 34 (1995) 1837.

4) N. F. Borrelli, D. W. Hall, H. J. Holland, and D. W. Smith: J. Appl. Phys. 61 (1987) 5399.

5) T. Yanagawa, Y. Sasaki, and H. Nakano: Appl. Phys. Lett. 54 (1989) 1495.

6) T. Yanagawa, H. Nakano, Y. Ishida, and K. Kubodera: Opt. Commun. 100 (1993) 118.

7) T. Miyoshi, Y. Makidera, T. Kawamura, N. Matsuo, and T. Kaneda: J. Lumin. 102-103 (2003) 614.

8) T. Miyoshi, H. Sera, N. Matsuo, and T. Kaneda: Jpn. J. Appl. Phys. 40 (2001) 2327. 


\section{Figure captions}

Fig. 1. Transmission spectra of unirradiated CdS-doped glasses, Y-02 and Y-44, and undoped glass, Y-0, at $300 \mathrm{~K}$.

Fig. 2. ESR spectra of X-ray-irradiated CdS-doped glasses, Y-02 and Y-44, at $300 \mathrm{~K}$. X-ray dose was $10 \mathrm{~Gy}$. The $g$ value of the signal at around $347 \mathrm{mT}$ is 2.01 , and that at around $351 \mathrm{mT}$ is 1.99 .

Fig. 3. Intensities of ESR signals of Y-02, Y-44, and the alanine dosimeter as a function of X-ray dose. Y-02(e) and Y-44(e) stand for the ESR signal at $g=1.99$, and Y-02(h) and Y-44(h) stand for the ESR signal at $g=2.01$. Lines were drawn through data points as visual guides.

Fig. 4. ESR spectra of X-ray-irradiated undoped glass, Y-0, at $300 \mathrm{~K}$. X-ray dose was 40 Gy. The $g$ value of the signal is 2.01 .

Fig. 5. Isochronal annealing results for X-ray-irradiated CdS-doped glasses, Y-02 and Y-44. X-ray dose was $10 \mathrm{~Gy}$. The annealing time was $15 \mathrm{~min}$. The normalized intensities of the ESR signals at $g=1.99$ are plotted as a function of annealing temperature. Curve was drawn through data points as a visual guide.

Fig. 6. Fading characteristics of X-ray-irradiated CdS-doped glasses, Y-02 and Y-44, and undoped glass, Y-0, at $300 \mathrm{~K}$. X-ray dose was $10 \mathrm{~Gy}$ for Y-02 and Y-44, and $40 \mathrm{~Gy}$ for Y-0. The intensities of the ESR signals at $g=1.99$ are plotted for Y-02 and Y-44, and those at $g=2.01$ are plotted for Y-0. The ESR intensities are normalized. The intensity ratios are as follows: 2 (Y-02), 1 (Y-44), and 0.07 (Y-0). Curves were drawn through data points as visual guides. 


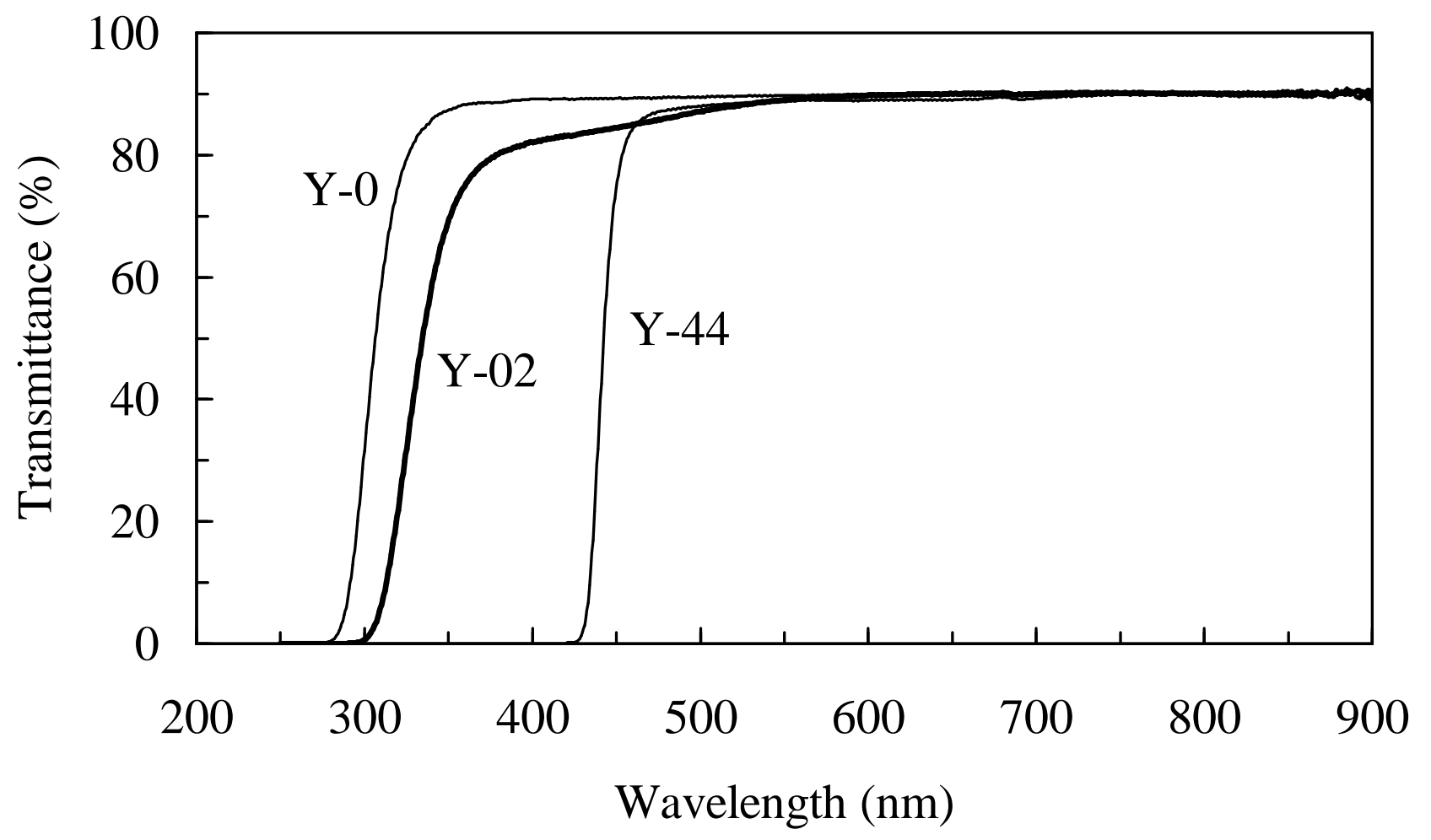

Fig. 1 


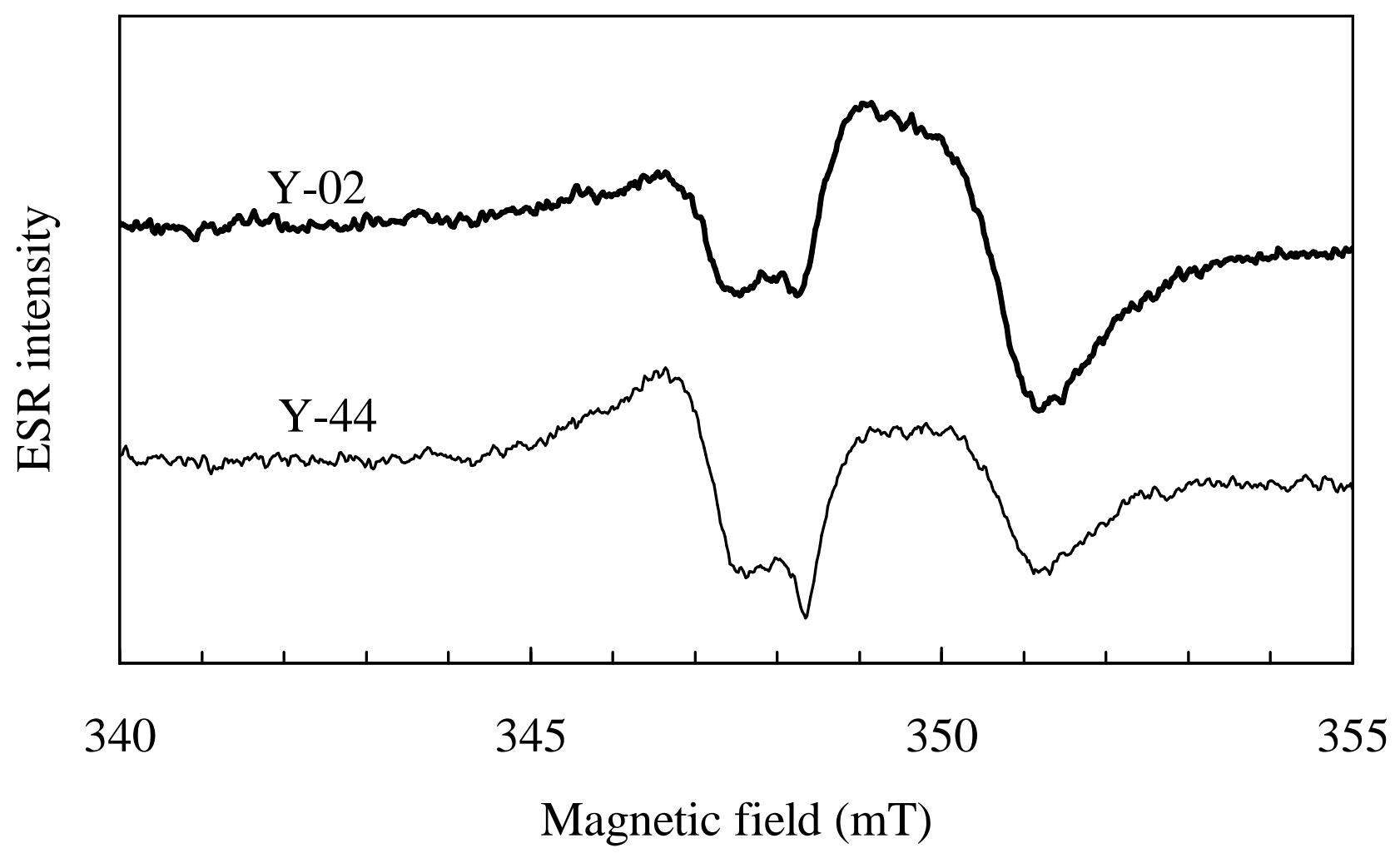

Fig. 2 


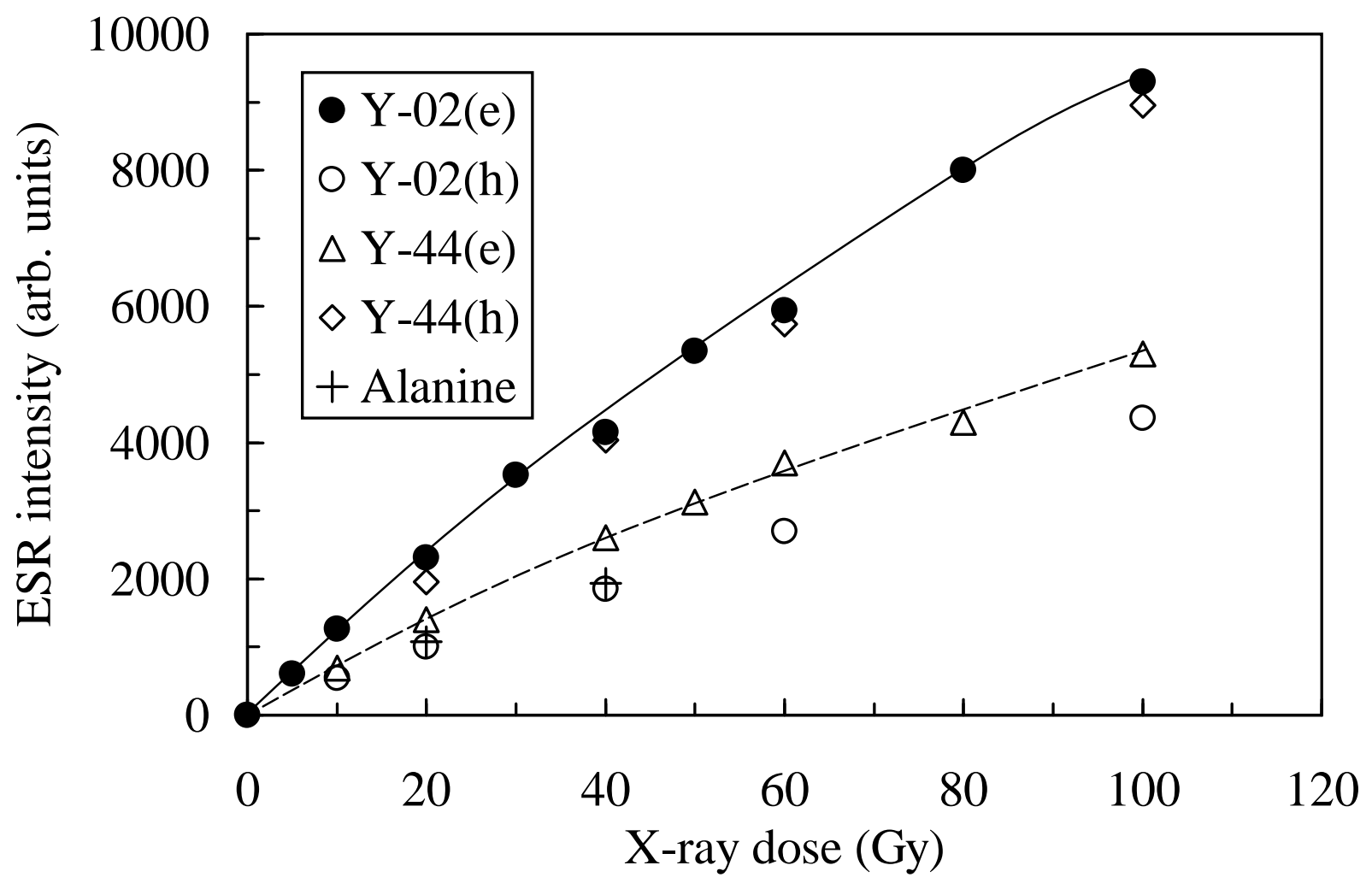

Fig. 3 


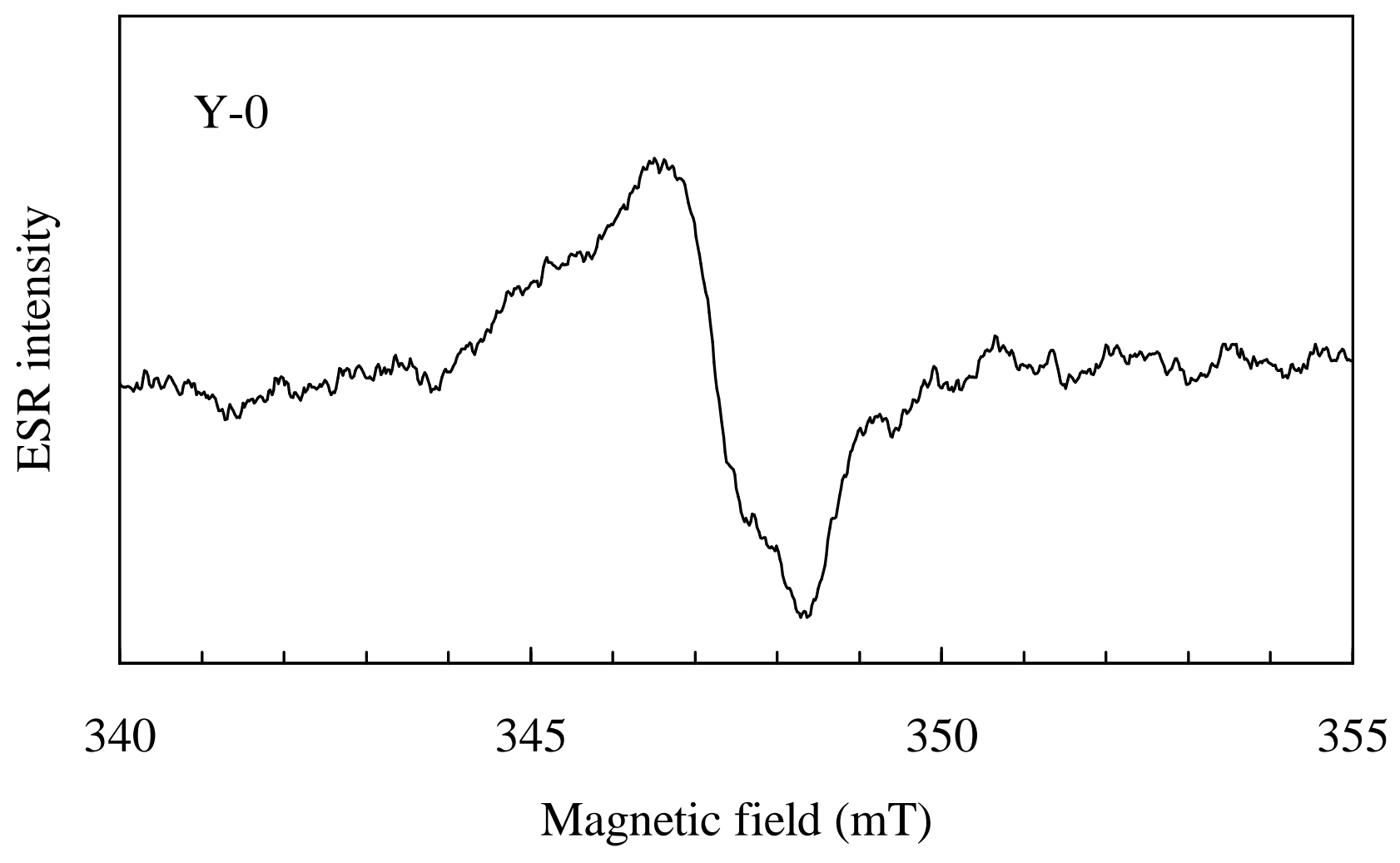

Fig. 4 


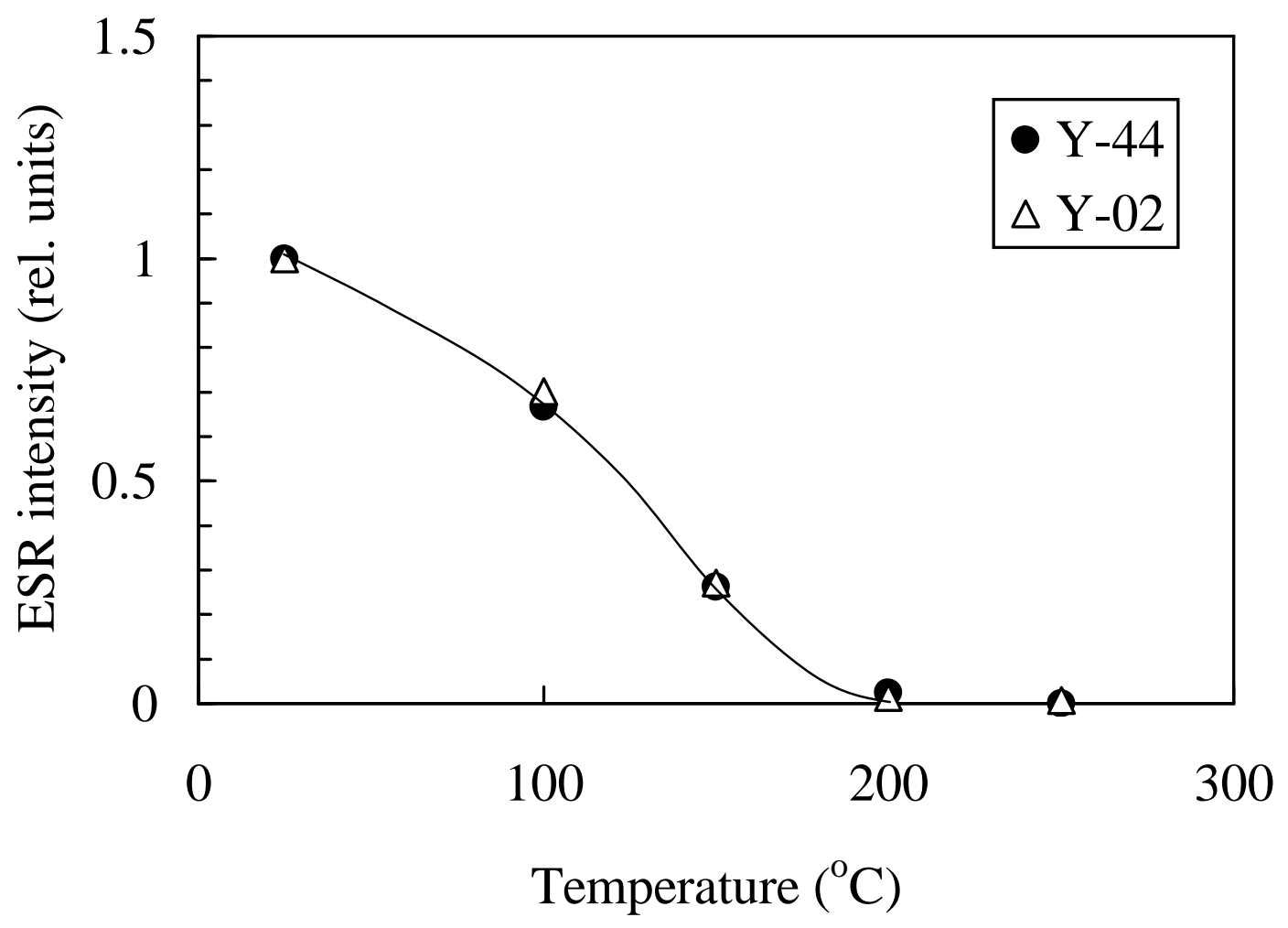

Fig. 5 


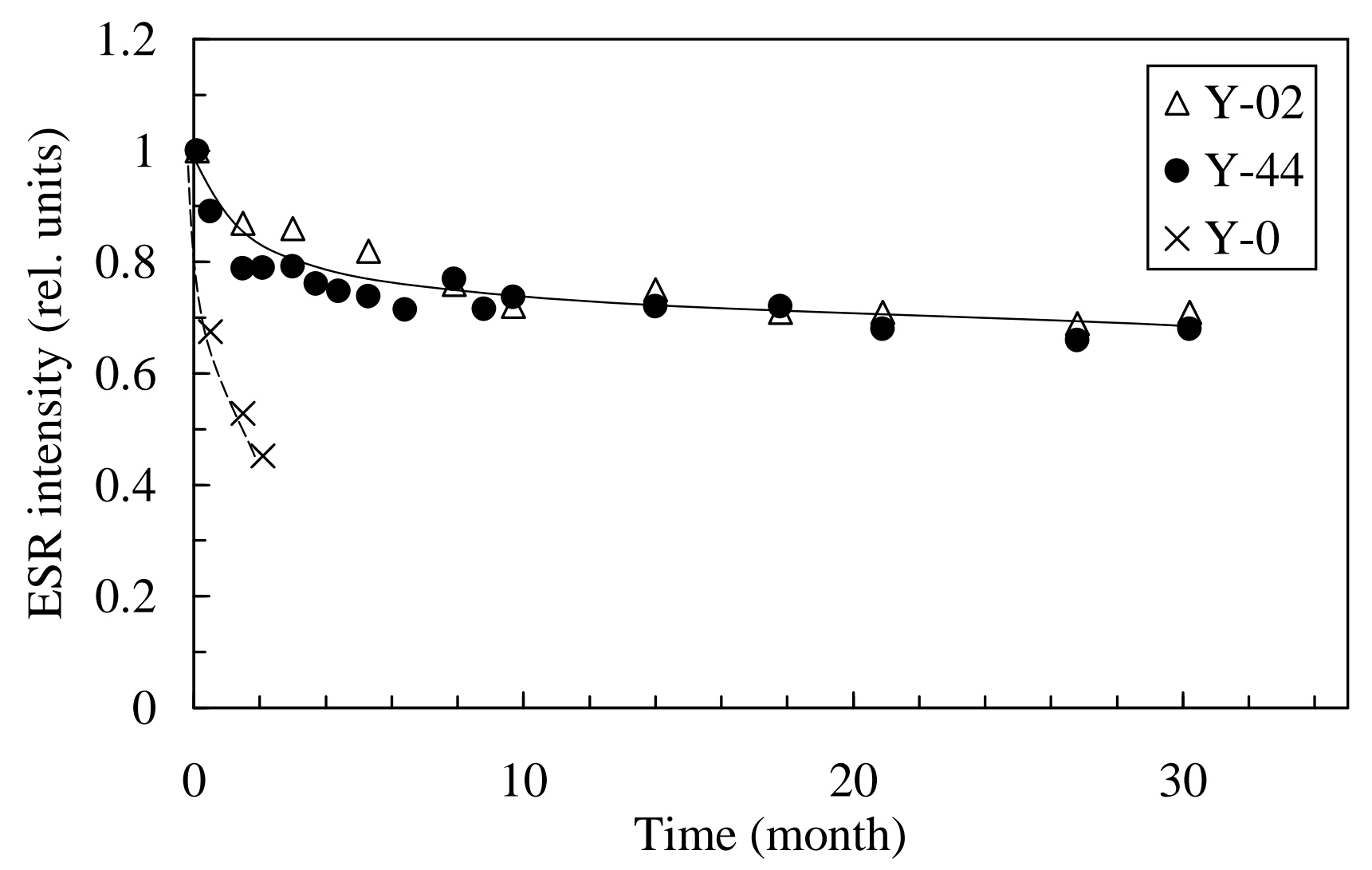

Fig. 6 